\title{
Nanoscale Mechanical Characterisation of Amyloid Fibrils Discovered in a Natural Adhesive
}

\author{
Anika S. Mostaert • Michael J. Higgins • \\ Takeshi Fukuma • Fabio Rindi • Suzanne P. Jarvis
}

Received: 23 June 2006 / Accepted: 7 August 2006 /

Published online: 5 October 2006

(C) Springer Science + Business Media B.V. 2006

\begin{abstract}
Using the atomic force microscope, we have investigated the nanoscale mechanical response of the attachment adhesive of the terrestrial alga Prasiola linearis (Prasiolales, Chlorophyta). We were able to locate and extend highly ordered mechanical structures directly from the natural adhesive matrix of the living plant. The in vivo mechanical response of the structured biopolymer often displayed the repetitive sawtooth force-extension characteristics of a material exhibiting high mechanical strength at the molecular level. Mechanical and histological evidence leads us to propose a mechanism for mechanical strength in our sample based on amyloid fibrils. These proteinaceous, pleated $\beta$-sheet complexes are usually associated with neurodegenerative diseases. However, we now conclude that the amyloid protein quaternary structures detected in our material should be considered as a possible generic mechanism for mechanical strength in natural adhesives.
\end{abstract}

Key words amyloid $\cdot$ natural adhesive $\cdot$ atomic force microscopy $\cdot$ adhesion $\cdot$ nanoscale mechanics $\cdot$ force measurements $\cdot$ extracellular polymeric substances $\cdot$ algae $\cdot$ biopolymer

\section{Introduction}

The ability of algae to adhere to a wide range of natural and artificial surfaces in marine, freshwater and terrestrial habitats is due to the secretion of extracellular polymeric substances (EPS) that have a diversity of chemical compositions [1]. The mechanism of adhesion typically involves initial, reversible attachment to a surface followed by an EPS secretion that forms a more permanent adhesive. Similar adhesive mechanisms are found

\footnotetext{
A. S. Mostaert $\cdot$ M. J. Higgins $\cdot$ T. Fukuma $\cdot$ S. P. Jarvis $(\square)$

Centre for Research on Adaptive Nanostructures and Nanodevices,

Trinity College Dublin, Dublin 2, Ireland

e-mail: suzi.jarvis@tcd.ie

F. Rindi

Department of Botany, Martin Ryan Institute, National University of Ireland, Galway, Ireland
} 
extensively in nature, not only in plants, but also invertebrate organisms such as barnacles and mussels.

Previously it has been explained how molecular level mechanical characteristics observed in adhesives, fibers and composites should contribute to their observed macroscopic mechanical strength, and, in the case of an adhesive, contribute to its success in permanently attaching the organism to its preferred substrate [2]. Specifically, Smith et al. [2] have described how particular mechanical benefit can be derived from a modular elongation mechanism.

Here, we investigate the nanoscale adhesive properties of a species of terrestrial algae, chosen because of its great ecological success in coping with environmental stress and its observed strong attachment to surfaces in its natural habitat and in culture. Prasiola linearis is a multicellular green macroalgal that occurs attached to grasses of saltmarshes and coastal lagoons, and is reported from localities of the Pacific North America region. P. linearis has a distinct adhesive holdfast structure located at the base of the differentiated plant which functions specifically for permanent adhesion. Using atomic force microscopy (AFM) we have directly probed the mechanical properties of the adhesive holdfast in vivo.

\section{Results and Discussion}

The AFM tip was brought into direct contact with the P. linearis EPS and both the approach and retraction of the tip was recorded. Most pulling measurements revealed force-extension profiles with irregularly spaced force peaks and plateaus, as seen in Figure 1. Such curves are typical of complex multi-molecular interactions involving the extension and breaking of loops and bonds and the peeling apart of the molecules between the tip and sample. However, the resulting force-extension curves at several EPS locations often revealed a highly regular sawtooth pattern of equally spaced peaks as shown in Figure 2. At these locations, sawtooth structures were typically seen in approximately $25 \%$ of the force curves, but in certain regions were present in $50 \%$ of the curves. The uniformity and reproducibility of the sawtooth pattern is particularly surprising given the chemical complexity of typical EPS. The mechanical response indicates that there is a highly ordered biopolymer exposed at the surface of the EPS matrix that consists of linked modular structural units of equal dimensions. Such biopolymers appear to be exposed at the surface in patches and are not distributed uniformly across the surface.

It has been explained previously that a repetitive sawtooth mechanical response on elongation is mechanically beneficial to a material under tension [2]. Smith et al. [2] highlight that this is regardless of the specific molecular composition and is due to the extra energy required to break the sacrificial bonds (typically within the folded modular units or loops) before exposing the backbone of the material to a high enough force to induce rupture. In our natural system, the repetitive and continual energy demands of trying to elongate adhesive material between the plant and the substrate probably accounts for the mechanical strength of the adhesive and consequently the ecological success of the plant.

To further elucidate the nature of the biopolymer within the EPS matrix we fitted the elastic response of the adhesion peaks using the worm-like chain model for semi-flexible polymers [3], as shown by the dashed lines in Figure 2b, d, e. A mean persistence length of $0.34 \pm 0.18 \mathrm{~nm}$ was found from a total of 100 curves. This length corresponds to building blocks of amino acids within a proteinaceous structure and is similar to those found for proteins such as titin, $0.4 \mathrm{~nm}[4,5]$, and tenascin, $0.42 \mathrm{~nm}[6]$. Many factors can affect the composition of EPS but algae are known to produce EPS, composed primarily of 
Figure 1 Nanoscale mechanical response of algal adhesive. (a),

(b) Examples of force-extension curves from the adhesive holdfasts of Prasiola linearis showing random adhesion of EPS components. Both the approach (grey; red in colour version) and retraction (black; blue in colour version) force traces are shown in each curve. (a) Several unbinding events are shown in this curve, and were typical for most force curves where sawtooths were not detected. (b) Often plateaus were seen in the force data indicating the peeling apart of molecules at constant force.
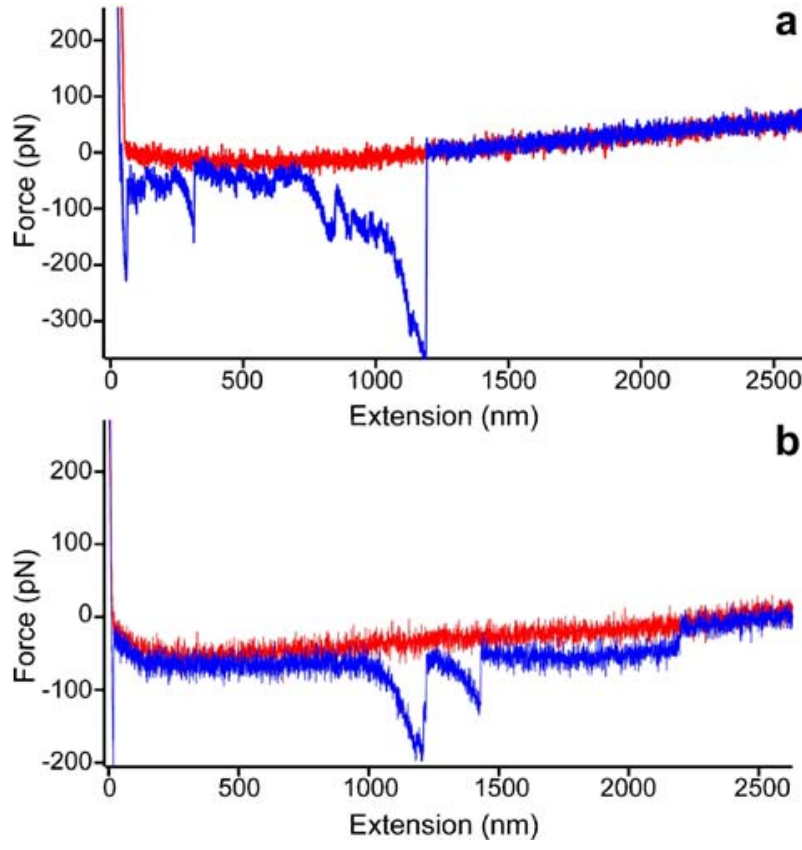
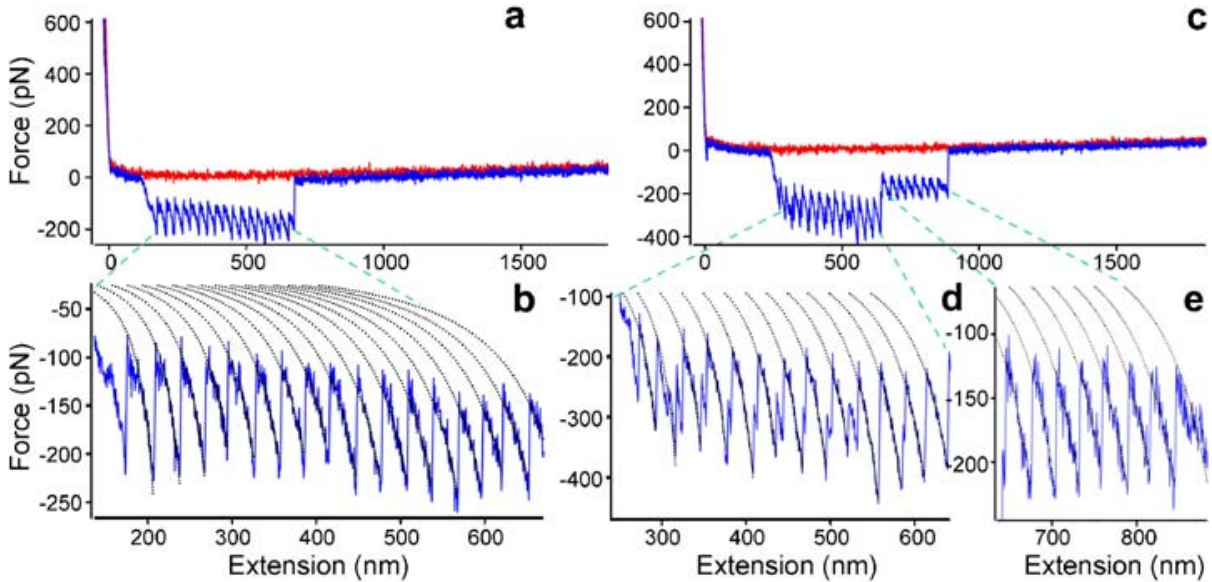

Figure 2 Nanoscale mechanical unfolding of amyloid fibril $\beta$-sheets in algal adhesive. (a-e), Representative force-extension curves from holdfasts of Prasiola linearis showing highly ordered sawtooth structures. (a) A mean persistence length of $0.22 \mathrm{~nm}$ was recorded for the 17 peaks observed in this particular curve. (b) Expansion of the sawtooth region of (a) shown fitted to the worm-like chain model (dotted lines). (c) Force-extension curve showing two sawtooth 'tiers'. (d), (e) Expansions of the two sawtooth regions are shown fitted to the worm-like chain model (dotted lines), one of which, (d), has a mean persistence length of $0.21 \mathrm{~nm}$ for the 13 peaks, and the other, (e), has a mean persistence length of $0.41 \mathrm{~nm}$ recorded for the eight peaks. 
polysaccharides with a small amount of protein and other components [7]. The presence of both polysaccharide and protein was confirmed in the EPS of P. linearis by histochemical staining. We also calculated a mean peak separation of $34.9 \pm 5.6 \mathrm{~nm}(n=1,463)$, which corresponds to the hidden length exposed on release of the sacrificial bonds within each module or unit within the structure. The average magnitude of the force peaks was found to be $244 \pm 36 \mathrm{pN}\left(2.5\right.$ to $\left.3.0 \mu \mathrm{m} \mathrm{s}^{-1}\right)$. This magnitude of force, at this extension rate, would be characteristic of that measured previously for systems containing hydrogen-bonded $\beta$ sheets [8].

For some curves, smaller persistence lengths were measured as shown in Figure 2a, where the persistence length was found to be $0.22 \mathrm{~nm}$. This indicates that, if the structure is proteinaceous, a highly ordered but parallel arrangement of proteinaceous entropic springs can readily occur between the tip and the sample. Springs in parallel increase the apparent overall stiffness of the material, and thus reduce the calculated persistence length [9]. In other curves, the average persistence length changed abruptly, and by a significant magnitude during elongation, an example of which can be seen in Figure 2c. We note that in such stepwise changes in persistence length, the subsequent average persistence length was always larger, implying that the biopolymer instantaneously reduces the number of proteinaceous springs within the structure when extended. In the case of the curve in Figure $2 \mathrm{c}$ the persistence length changes from $0.21 \mathrm{~nm}$ (Figure 2d) to $0.41 \mathrm{~nm}$ (Figure 2e).

To identify the composition of this structure, which would appear to be proteinaceous, directly during the AFM elongation measurements, is practically impossible. Direct imaging of the EPS at high resolution is extremely difficult due to the heterogeneous and adhesive nature of EPS, and even if successful would not provide sufficiently detailed chemical information to uniquely identify the structure.

Sequencing of the constituent proteins of natural adhesives has also proved highly problematic due to the difficulties of separating out the proteins [10], and, specifically in the case of algae, separating proteins from other EPS components such as polysaccharides. However, there is no evidence to suggest that the mechanical response we measure here is even attributable to one particular protein and its tertiary structure. Given the time and effort involved in sequencing it would seem prudent to establish any possible contribution of quaternary protein structures before embarking on such a route.

It has been established recently that isolated, model amyloid fibrils under a tensile axial loading force can also produce a sawtooth mechanical response if the fibril is pulled at some point along its length such that the individual proteins or peptides are unravelled sequentially from the bulk of the fibril [11]. If, however, the protein or peptide molecules on the fibril surface are pulled close to the end of the fibril, or the fibril is damaged under the tip so as to create two ends, then the constituent proteins or peptides remain as a continuous intermolecular $\beta$-sheet which is peeled from the bulk of the fibril, a resulting in a force plateau [11].

Thus we considered, in particular, the possible presence of amyloid quaternary protein structures in our material. These fibrilar structures are $\beta$-sheet-rich and highly ordered. They do not appear to depend on a specific amino acid sequence although certain proteins are considered to be more likely to form amyloid fibrils than others in vivo. These so-called amyloidogenic proteins have been found to be ubiquitous in nature [12]. Recent evidence suggests that amyloid fibrils can readily self-assemble from most polypeptides under appropriate conditions, which are usually slightly denaturing [13]. This is because denaturing a protein from its usual tertiary structure increases the probability of it misfolding into the amyloid fold. The local environments of many organisms attached via a natural adhesive could be considered denaturing due to $\mathrm{pH}$ or dehydration. 
To investigate the possible presence of amyloid structures in our algal adhesive we used the amyloid-selective dyes Congo red and thioflavin- $T$ that preferentially bind to amyloid [14]. The characteristic green-gold birefringence observed with cross-polarized light after staining with Congo red dye provides evidence that indeed amyloid structures are present and can be readily detected in our material (Figure 3d). It is particularly interesting to note that the amyloid fibrils are detected close to the attached end of the holdfast and appear to be extracellular. The local specificity of the response reduces the likelihood that the signal is a false positive. Due to the expected autofluorescence of our material we also investigated a control sample without Congo red. This was found to give a faint signal under cross-polarized light that has been included for comparison (Figure 3b). Direct binding to amyloid structures with the fluorochrome dye thioflavin-T was detected with confocal microscopy and spectral imaging (Figure 4). The EPS coating of the plant gave a signal for thioflavin-T (Figure 4a), but more notable was that the adhesive around the base of the plant only produced a signal for thioflavin-T, with no autofluorescent signal (Figure $4 \mathrm{~b}, \mathrm{c}$ ). The specificity and sensitivity of Congo red and thioflavin-T as amyloidspecific dyes have been questioned despite their extensive use for characterising the presence of amyloid fibrils and their rates of formation [14]. However, here in combination they provide evidence for the presence and spatial distribution of amyloid in our algal adhesive.

Bright field
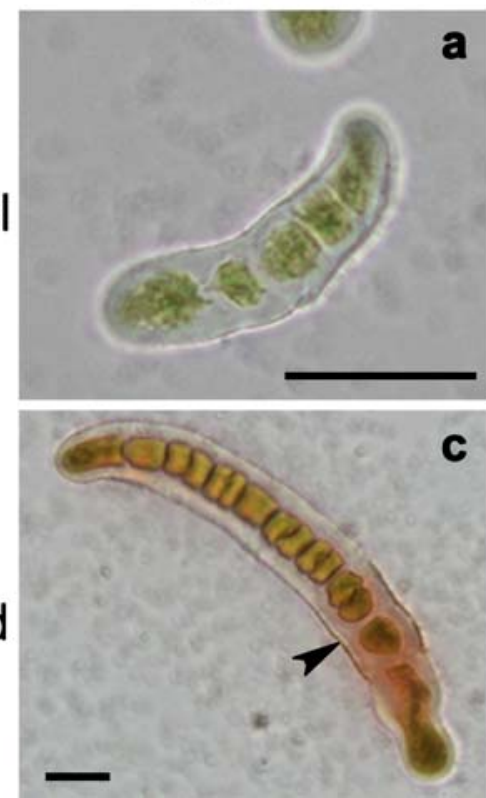

Polarized light
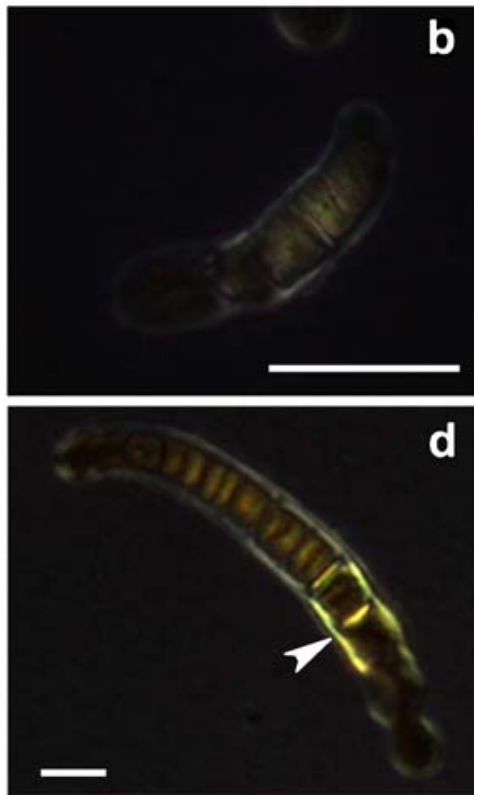

Figure 3 Evidence of amyloid in Prasiola linearis adhesive as indicated by Congo red birefringence. Unstained control plant attached to glass surface is shown with (a) bright field and (b) cross-polarized light showing faint autofluorescence. (c), (d) Plant treated directly with Congo red dye. (c) Bright field microscopy showing binding of the dye in the adhesive region of the algal frond (arrow head), and (d) the same region showing green-gold birefringence under cross-polarized light (arrow head) characteristic of amyloid deposits. Scale bars $=25 \mu \mathrm{m}$. 

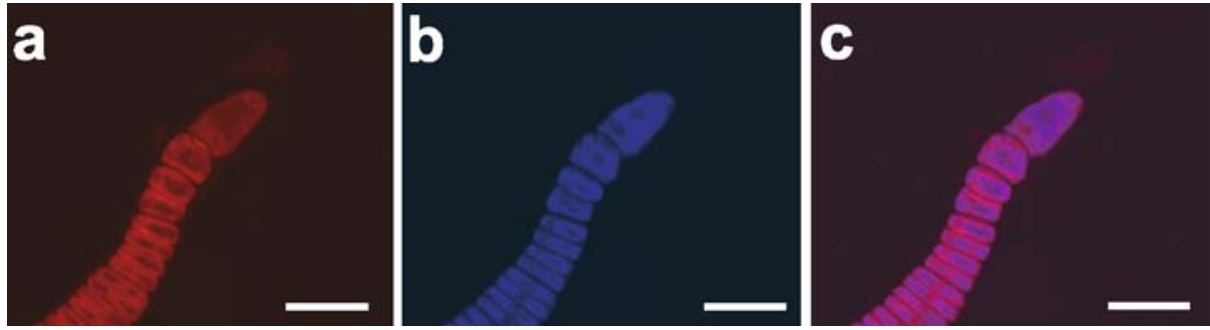

Figure 4 Direct binding of thioflavin- $T$ to $\beta$-sheet structures within the secreted extracellular polymeric substances of Prasiola linearis. Plants attached to glass coverslips were treated directly with thioflavin-T. Zeiss confocal Meta microscopy allows separation of the emission of the thioflavin-T bound to $\beta$-sheet structures (a), and the algal autofluorescence (b). The adhesive around the base of the plant only produced a signal for thioflavin-T (c). Scale bars $=20 \mu \mathrm{m}$.

Amyloid fibrils are known to form from proteins misfolding into intramolecular $\beta$-sheets and self-assembling into highly regular intermolecular crossed $\beta$-sheets structures [15]. These intermolecular $\beta$-sheets assemble into protofilaments that combine to form fibrils [15]. If the AFM tip attaches, via non-specific adhesion, to a fibril somewhere along its length, and is then pulled away, many of the bonds connecting the exposed intermolecular $\beta$-sheet to the one below are being pulled in parallel. This strongly pins the $\beta$-sheet to the bulk of the fibril and thus it is possible that the applied tensile force can become sufficiently large for successive misfolded proteins within the intermolecular $\beta$-sheet to unravel sequentially as shown schematically in Figure 5. This sequential unfolding of individual molecules corresponds to the peaks in our force-extension curves. The high number of unfolding events implies a strong interaction between each of the proteins within the intermolecular $\beta$-sheet, i.e., cross-linking. Thus the peak separation $(34.9 \mathrm{~nm})$ corresponds approximately to the length of the amino-acid chain between cross-linking of the protein to those on either side. This indicates that the proteins in P. linearis that form the fibrils are likely to be relatively small proteins of the order of 100-150 amino acids in length. In our model, an unphysically small persistence length may be observed if more than one intermolecular $\beta$-sheet is attached to the tip.

Considering the mechanical manipulation of $\beta$-sheets (or multiple layers thereof) within a quaternary amyloid protein structure provides a simple mechanism that allows for the observed sawtooth mechanical response and amino acid persistence length correspondence as well as the short persistence lengths sometimes observed in our measurements.

The amyloid fibril structure is a very stable form for proteins to adopt and we suggest, based on our measurements, that the misfolding of protein into amyloid fibrils would have been mechanically beneficial within the adhesive mucilage of $P$. linearis and thus has been evolutionarily conserved. It would appear that this native amyloid does not have any detrimental effect on the organism due to its presence in the adhesive from an early stage in the plant's growth.

Experiments indicate that amyloid formation from a protein with a stable monomeric structure does not necessitate that the entire protein participates in the amyloid core structure [16]. Thus additional protein domains that may contain adhesive residues could retain their native function external to the fibril core structure. It is also possible that this type of native amyloid (termed amyloidin [17]) may provide a generic mechanism for mechanical strength in other natural adhesives and natural materials, in other words a generic mechanical amyloidin. 


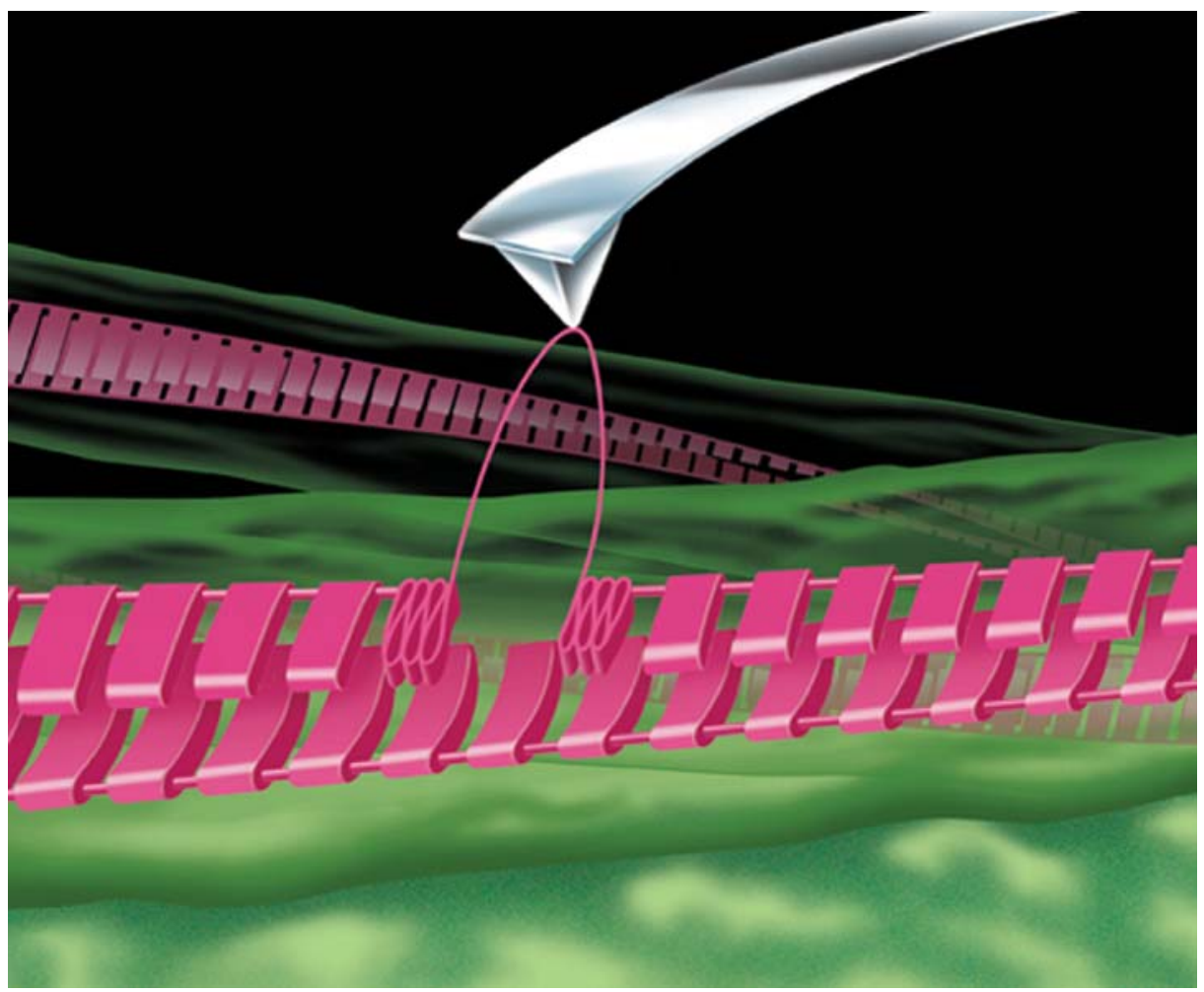

Figure 5 Schematic model of the mechanical manipulation of a single intermolecular $\beta$-sheet (pink) at the surface of an amyloid fibril (green) by an atomic force microscope tip. The schematic is not intended to suggest a specific molecular structure within the fibril. Molecular level structural data on amyloid fibrils formed from proteins, as opposed to peptide fragments, remains scarce. The schematic shows interacting molecular building blocks of intramolecular $\beta$-sheets running perpendicular to the direction of the fibril making up extended intermolecular $\beta$-sheets that form the fibril. The 'hidden length' is contained within the folds of each individual molecule (detail is shown only below the tip) and the 'sacrificial bonds' exist between the folds. Interactions between the molecular building blocks are significantly stronger than these sacrificial bonds and imply cross-linking between each protein, as indicated with the solid line running along the back of the manipulated intermolecular $\beta$-sheet.

\section{Experimental Procedures}

\subsection{Algal collection and culture}

P. linearis was collected from Argyl Lagoon, St. Juan Island, Washington State, USA, where it occurred attached to the stems of Salicornia grasses. The unialgal culture was maintained in von Stosch Enriched Seawater [18] at $10^{\circ} \mathrm{C}, 16: 8 \mathrm{~h}$ light:dark cycle, 40 $45 \mu \mathrm{mol}$ photons per square meter per second of illumination.

\subsection{Attached algae for AFM}

Sections of fertile fronds of $P$. linearis were placed into small dishes containing a glass coverslip and $20 \mathrm{ml}$ of culture media. Within five days of observing release of aplanospores 
from parent plants, the coverslips with attached spores and young sporelings were glued onto glass slides for AFM investigation. This species exhibited strong and rapid attachment to the coverslips making it an ideal organism for AFM where whole cells must be immobilized.

\subsection{Histochemical staining}

The presence of polysaccharide and protein using Stains-All (Sigma) for protein and Alcian Blue (Sigma) for polysaccharide (data not shown) were confirmed in natural adhesive of plants attached to glass coverslips (as described for AFM above).

Attached plants on glass coverslips were also treated directly with $0.5 \%$ Congo red (Sigma) in 50\% ethanol [14] for $3 \mathrm{~min}$, before being washed with deionized water and mounted in glycerol gelatin (Sigma). Specimens were viewed immediately at $40 \times$ magnification with a Zeiss AxioCam 200 microscope fitted with a polarizer and analyser for switching from bright field imaging to polarizing microscopy. Digitized images were obtained using an AxioCam HR colour camera.

Confocal microscopy was used in order to use the fluorophore thioflavin-T (Sigma) for the detection of amyloid structures in the EPS of P. linearis. A Zeiss confocal Meta system was used to allow separation of multiple fluorophores based on their emission spectra. The algal autofluorescence emission was determined by exciting an unstained control sample at $488 \mathrm{~nm}$ and collecting the emission at 505 to $620 \mathrm{~nm}$. Emission of thioflavin-T binding to amyloid structures was determined using a control sample of amyloid fibrils grown from a synthesized amyloid $\beta$-Protein $25-35$ (H-1192, lot no. 0568384, Bachem, UK). The peptide was prepared as $1 \mathrm{mM}$ in water, and fibrils formed within one week at room temperature (verified by Congo red birefringence). A sample was deposited onto a glass slide, stained with thioflavin-T (10 $\mu \mathrm{m}$ aqueous solution for $5 \mathrm{~min})$, and emission collected in the same way as for the unstained algal control. Binding of algae by thioflavin-T was detected by directly staining plants attached to coverslips with $10 \mu \mathrm{m}$ aqueous thioflavin- $\mathrm{T}$ for $5 \mathrm{~min}$ before being washed in deionized water. Thioflavin-T stained algae were also excited at $488 \mathrm{~nm}$ and emission collected at 505 to $620 \mathrm{~nm}$. Using the two control emission spectra, it was possible to subtract the alga's autofluorescence from the amyloid fibrils stained with thioflavin-T.

AFM of natural adhesive AFM force measurements were made in Milli-Q (Millipore) ultrapure water using an Asylum MFP-3D AFM (Asylum Research, Santa Barbara, CA) mounted on top of an Olympus IX51 inverted optical microscope for visualizing and manually positioning regions to be probed. Force measurements were taken in static mode at a rate of $0.5 \mathrm{~Hz}\left(2.5\right.$ to $3.0 \mu \mathrm{m} \mathrm{s}^{-1}$ ), using $\mathrm{Si}_{3} \mathrm{Ni}_{4}$ cantilevers (CSC38 series, MikroMasch) with spring constants between 0.13 and $0.15 \mathrm{~N} \mathrm{~m}^{-1}$ calibrated using the Sader Method [19]. IGOR PRO (Wavemetrics, OR) data analysis software was used to analyze the force measurements. Measurements were made directly on the exposed region of the adhesive holdfasts observed at the base of differentiated sporelings of $P$. linearis.

\footnotetext{
Acknowledgments We thank Dr Aoife McLysaght, Trinity College Dublin, Ireland, for fruitful discussions; Dr Sean Callanan and his colleagues, University College Dublin, Ireland, for histochemical staining advice; Dr Paula Maguire, Trinity College Dublin, Ireland, for assistance with confocal microscopy and analysis; and Professor Louis Hanic and Dr Sandra Lindstrom, University of British Columbia, Canada, for providing the original collection of algal material. This work was funded by Science Foundation Ireland (01/PI.2/C033).
} 


\section{References}

1. Fletcher, R., Callow, M.E.: Settlement, attachment and establishment of marine algal spores. Br. Phycol. J. 27, 303-329 (1992)

2. Smith, B.L., Schäffer, T.E., Viani, M., Thompson, J.B., Frederick, N.A., Kindt, J., Belcher, A., Stucky, G.D., Morse, D.E., Hansma, P.K.: Molecular mechanistic origin of the toughness of natural adhesives, fibres and composites. Nature 399, 761-763 (1999)

3. Bustamante, C., Marko, J.F., Siggia, E.D., Smith, S.: Entropic elasticity of $\lambda$-phage DNA. Science 265, 1599-1600 (1994)

4. Rief, M., Gautel, M., Oesterhelt, F., Fernandez, J.M., Gaub, H.E.: Reversible unfolding of individual titin immunoglobulin domains by AFM. Science 276, 1109-1112 (1997)

5. Tskhovrebova, L., Trinick, J., Sleep, J.A., Simmons, R.M.: Elasticity and unfolding of single molecules of the giant muscle protein titin. Nature 387, 308-312 (1997)

6. Oberhauser, A.F., Marszalek, P.E., Erickson, H.P., Fernandez, J.M.: The molecular elasticity of the extracellular matrix protein tenascin. Nature 393, 181-185 (1998)

7. Wetherbee, R., Lind, J.L., Burke, J., Quatrano, R.S.: The first kiss: establishment and control of initial adhesion by raphid diatoms. J. Phycol. 34, 9-15 (1998)

8. Brockwell, D.J., Beddard, G.S., Paci, E., West, D.K., Olmsted, P.D., Smith, D.A., Radford, S.E.: Mechanically unfolding the small, topologically simple protein L. Biophys. J. 89, 506-519 (2005)

9. Bemis, J.E., Akhremitchev, B.B., Walker, G.C.: Single polymer chain elongation by atomic force microscopy. Langmuir 15, 2799-2805 (1999)

10. Waite, J.H., Qin, X.: Polyphosphoprotein from the adhesive pads of Mytilus edulis. Biochemistry 40, 2887-2893 (2001)

11. Fukuma, T., Mostaert, A.S., Jarvis, S.P.: Explanation for the mechanical strength of amyloid fibrils. Tribol. Lett. (2006), dx.doi.org/10.1007/s11249-006-9086-8.

12. Bateman, A., Lachlan, C., Durbin, R., Finn, R.D., Volker, H., Griffiths-Jones, S., Khanna, A., Marshall, M., Moxon, S., Sonnhammer, E.L.L., Studholme, D.J., Yeats, C., Eddy, S.R.: The Pfam protein families database. Nucleic Acids Res. (Database issue) 32, D138-D141 (2004)

13. Dobson, C.M.: Protein folding and misfolding. Nature 426, 884-890 (2003)

14. Vowles, G.H., Francis, R.J.: Amyloid. In: Bancroft, J.D., Gamble, M. (eds.) Theory and Practice of Histological Techniques, 5th ed., pp. 303-324. Churchill Livingstone, Harcourt, London, UK (2002)

15. Sipe, J.D.: Amyloid Proteins: The Beta Sheet Conformation and Disease. Wiley, Weinheim, Germany (2005)

16. Baxa, U., Speransky, V., Stevens, A.C., Wickner, R.B.: Mechanism of inactivation on prion conversion of the Saccharomyces cerevisiae Ure2 protein. Proc. Natl Acad. Sci. U.S.A. 99, 5253-5260 (2002)

17. Fowler, D.M., Koulov, A.V., Alory-Jost, C., Marks, M.S., Balch, W.E., Kelly, J.W.: Functional Amyloid formation within mammalian tissue. PLoS Biol. 4, 100-107 (2006)

18. Guiry, M.D., Cunningham, E.M.: Photoperiodic and temperature responses in the reproduction of northeastern Atlantic Gigartina acicularis (Rhodophyta: Gigartinales). Phycologia 23, 357-367 (1984)

19. Sader, J.E., Chon, J.W.M., Mulvaney, P.: Calibration of rectangular atomic force microscope cantilevers. Rev. Sci. Instrum. 70, 3967-3969 (1999) 